\title{
Image-based Stress Field Reconstruction in Complex Media
}

\author{
R. Seghir ${ }^{1 \mathrm{a}}$, F. Pierron ${ }^{2}$ and L. Fletcher ${ }^{2}$ \\ ${ }^{1}$ Research Institute in Civil and Mechanical Engineering (GeM), Ecole Centrale de Nantes, Université de Nantes, UMR \\ 6183 CNRS, F-44 321 Nantes, FR \\ ${ }^{2}$ Engineering and the Environment, University of Southampton, Highfield campus, University Road, Southampton \\ SO17 1BJ, UK
}

\begin{abstract}
In many instances in life, materials around or within us suffer deformation at high rates. This is the case when engineering structures undergo e.g. impact, crash, forming or pulsed welding. Another important area concerns biological tissues injuries. In those contexts, the transience and inhomogeneous aspect of such loadings as well as the strong multi-physic couplings induced by quasi-adiabatic conditions make both: the experimental capture of the mechanical response very challenging and all the potential assumptions regarding the constitutive relation of the deforming material extremely hazardous. To overcome both issues, we demonstrate in the following that experimental full-field measurements of accelerations can be directly used to inverse the local equilibrium equation and reconstruct fields of stress tensor with no assumption at all on the constitutive relation and its potential spatial and temporal variations. It is also demonstrated that both experimental stress and strain fields can be recombined to eventually identify the local tangent stiffness tensor of the material. The latest constitutes a first step in the field of "model identification", as opposed to parameters' model identification.
\end{abstract}

Keywords: ultra-high speed imaging, dynamic, stress, model identification

\section{Introduction}

When localized deformation forms in a material, due to the loading e.g. dynamic waves, to instabilities e.g. necking, adiabatic shear-bands or simply when cracking, assumptions or models are required to connect experimental measurements at the VER scale and the true local (thermo-) mechanical state. Even if the last 30 years have been characterized by a massive expansion of field measurement techniques, such models still cannot be validated at the scale there are formulated since local stresses still are inaccessible. Furthermore, in many cases only energy quantities are sought as in fracture mechanics where the fracture energy could be simply derived from a local energy balance regardless of the constitutive equation if stress and strain fields were accessible. In that context, recent developments in dynamic optical metrology have shown that acceleration fields can be experimentally captured [1], then, using dedicated test configuration such as inertial impact test, the later can be used as an image-embedded load cell if the material density is known [2], allowing a contactless measurement of both averaged strain and stress. More recently, such a methodology has allowed capturing a variety of local dynamic stress-strain responses within PMMA specimens subjected to unidirectional gradients of temperature and strain-rate [3]. It has confirmed the ability of the technique to quantitatively capture the local mechanical response variations under a 1D loading condition. In the following, we extend the concept to full-field stress tensor reconstruction. Explicit dynamic simulations are used as proof of concept, then components of the stress fields are recovered under plane stress assumptions and material stiffness maps are back calculated recombining the "measured" strain and the identified stress. While only elastic, homogeneous simulations are used in the following, the methodology is generic and independent of the material behavior and more important directly applicable to experiments where ultra-high speed imaging are used.

\section{Framework}

The main idea behind the proposed "non-parametric" stress reconstruction methodology is to replace a standard a priori parametrization of the material "law" by a piecewise spatial parametrization of the acceleration and by extension of the stress components through the equilibrium. The latest is eventually completed with the very general rules that a field of stress tensor has to satisfy regardless of its constitutive equation, i.e. conservation laws, linear and angular momentum, boundary conditions and continuity. In the following, acceleration fields are chosen bi-linear per element leading to a quadratic form of the piecewise sought components of the stress tensor field. As a consequence, the equilibrium 
provides 6 independent equations per elements, imposing cross-slopes and curvatures, continuity provides 18 independent equations, connecting elements state, and boundary conditions provide the constants. Finally, an overdetermined linear system of equation is assembled and can be solve in a least-square sense if acceleration fields, material density and boundary conditions are measured. In the following, the material density is considered homogeneous and reasonably independent from rates and temperature, acceleration fields are obtained by double differentiation of displacements output from explicit dynamic simulations (but currently available experimentally [2]), and boundary conditions are partially known due the test configuration. Indeed, we focus here on an inertial impact test configuration [2] where the free boundary conditions impose the normal and the tangent components of the stress tensor to be zero at all times at the specimen borders. Unknown boundary conditions, i.e. at impact edge, are eventually recovered by iteratively back identifying the specimen tangent stiffness map and updating local stresses components from strains

\section{Simulation}

The model consists in a $54 \mathrm{~mm} \times 34 \mathrm{~mm}$ rectangular specimen, meshed with $136 \times 216$-nodes quadrilateral plan stress elements. The loading is introduced via a XX ns and XX N uniform pulse at the impact edge. The considered material is isotropic linear elastic and homogeneous and properties are chosen as follows: $\rho=14000 \mathrm{~kg} \cdot \mathrm{m}^{-3}, Q_{11}=$ $580 \mathrm{GPa}, Q_{12}=140 \mathrm{GPa}, G_{12}=210 \mathrm{GPa}$. The explicit dynamic simulation is performed under LS-Dyna and results are output with a time-step of $200 \mathrm{~ns}$, i.e. equivalent to an acquisition rate of $5 \mathrm{MHz}$. It is worth noticing that while the material is homogeneous, stress fields are neither uniform nor constant or uniaxial. Indeed the loading produces waves crossing back and forth into the sample and interfering each other. For the mesh reconstruction, a mesh size has to be chosen. The finer the mesh the better the stress reconstruction. Nevertheless, elements have to be large enough to identify the set of parameters, here 5 per stress component. Hence, in the following a stress mesh size of $5 \times 5$ FE elements is chosen.

\section{Results}

For a sake of clarity, Figure 1 a) and b) present in a first place the error, mean and standard deviation over the field, on the reconstructed stress and tangent stiffness components at a specific time step, $t=16 \mu \mathrm{s}$. Figure $1 \mathrm{c})$ presents the associated equilibrium gap along the $x$ and $y$ axis, i.e. the different between the acceleration fields time the density and the divergence of the reconstructed stress tensor fields. Notice that in the following, identification windows of $15 \times 15$ FE elements $(3 \times 3$ stress elements $)$ are used to back identify the tangent stiffness maps. Iterations correspond to successive updates of the boundary conditions starting from a fully free-standing specimen assumption.
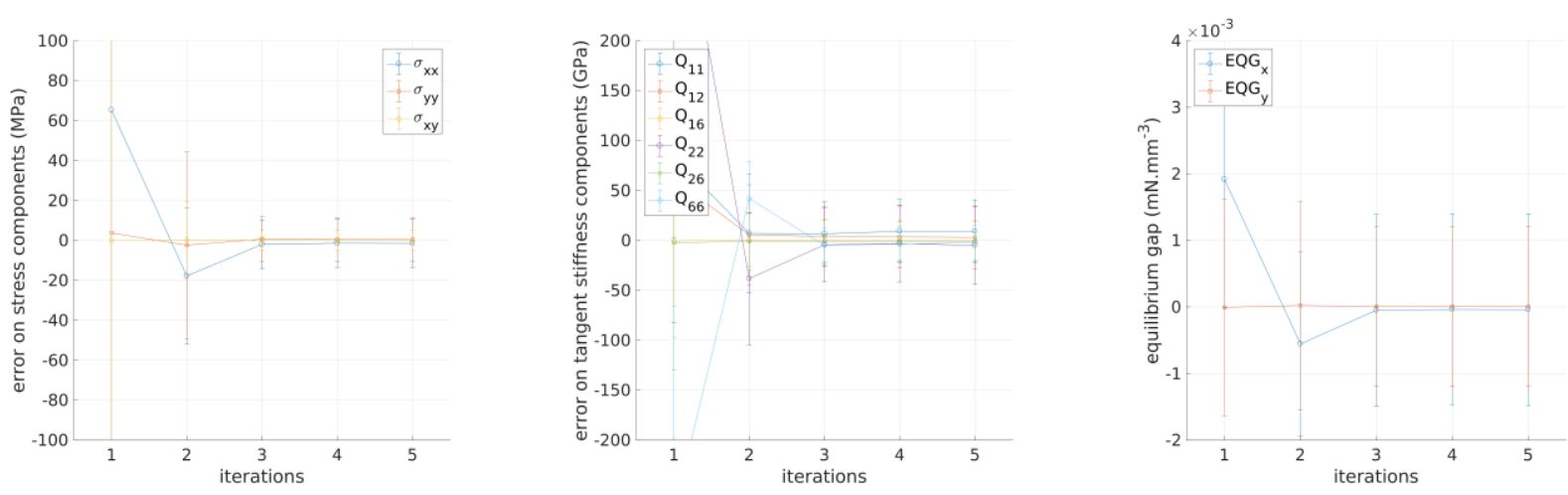

Figure 1: identification error for a stress mesh of $5 \times 5 \mathrm{FE}$ elements at time $t=16 \mu \mathrm{s}$. a) Error on stress, b) on tangent stiffness and c) associated equilibrium gap.

The average value of the equilibrium gaps evidence that the reconstructed stress tensor field well balance the acceleration forces at every stress elements. The standard deviation is here directly dependent on the parametrization of the acceleration field. Indeed, only bi-linear variations over a $5 \times 5 \mathrm{FE}$ element can be captured nevertheless its remains neglegible compared to the signal dynamic, about $10^{5} \mathrm{mN} \cdot \mathrm{mm}^{-3}$. Both results confirms that the reconstructed stress tensor fields are fully statically admissibles. Then, while a massive standard deviation is observed on reconstructed stress components at the first calcul iteration, i.e. free-standing assumptions, the errors rapidally decrease and reach $-1^{ \pm 12} \mathrm{MPa}, 0^{ \pm 11} \mathrm{MPa}$ and $0^{ \pm 5} \mathrm{MPa}$ for the $\sigma_{x x}, \sigma_{y y}$ and $\sigma_{x y}$ respectively, i.e. less than $2 \%$ of the signal dynamic. Similarily, tangent stiffness components converge rapidely and the errors reach $2^{ \pm 5} \%, 1^{ \pm 6} \%, 2^{ \pm 22} \%, 1^{ \pm 8} \%$ for $Q_{11}, Q_{22}, Q_{12}$ and $Q_{33}$ respectivelly. $Q_{13}$ and $Q_{23}$ are found to be equal to $0^{ \pm 20} \mathrm{GPa}$. The average behavior is perfectly captured, within $2 \%$ of error, and the material symmetry is well captured. Locally, $5 \%$ variations are found on the more active parameters (loading mainly unidirectional), but $20 \%$ error is found on quasi-null or poorly active parameters. It is important to notice that any a priori knownledge on the material symmetry would significanlty decrease the uncertainty. Indeed, here the 6 components are treated as fully independant. An other solution would be to enlarge the identification 
window until finding the good compromise between the desired spatial resolution of the material properties fields and their accuracy.

Then, figure $2 \mathrm{a}-\mathrm{c})$ presents converged ( $5^{\text {th }}$ iteration) stress fields (same time step as in Fig 1) as well as, b) the comparison between FE and reconstructed stresses over time at a specific point (marked with a white dot) and finally the variability over time and space of the identified material properties (mean and standard deviation). Fields evidence the great heterogeneity of the stress components and subfigure d) shows the ability of the proposed methodology to accuralty capture its time variations. Plot e) shows that material properties are reasonably well captured over time. Some time step lead to higher error due to the decrease in the dynamic of the signal.

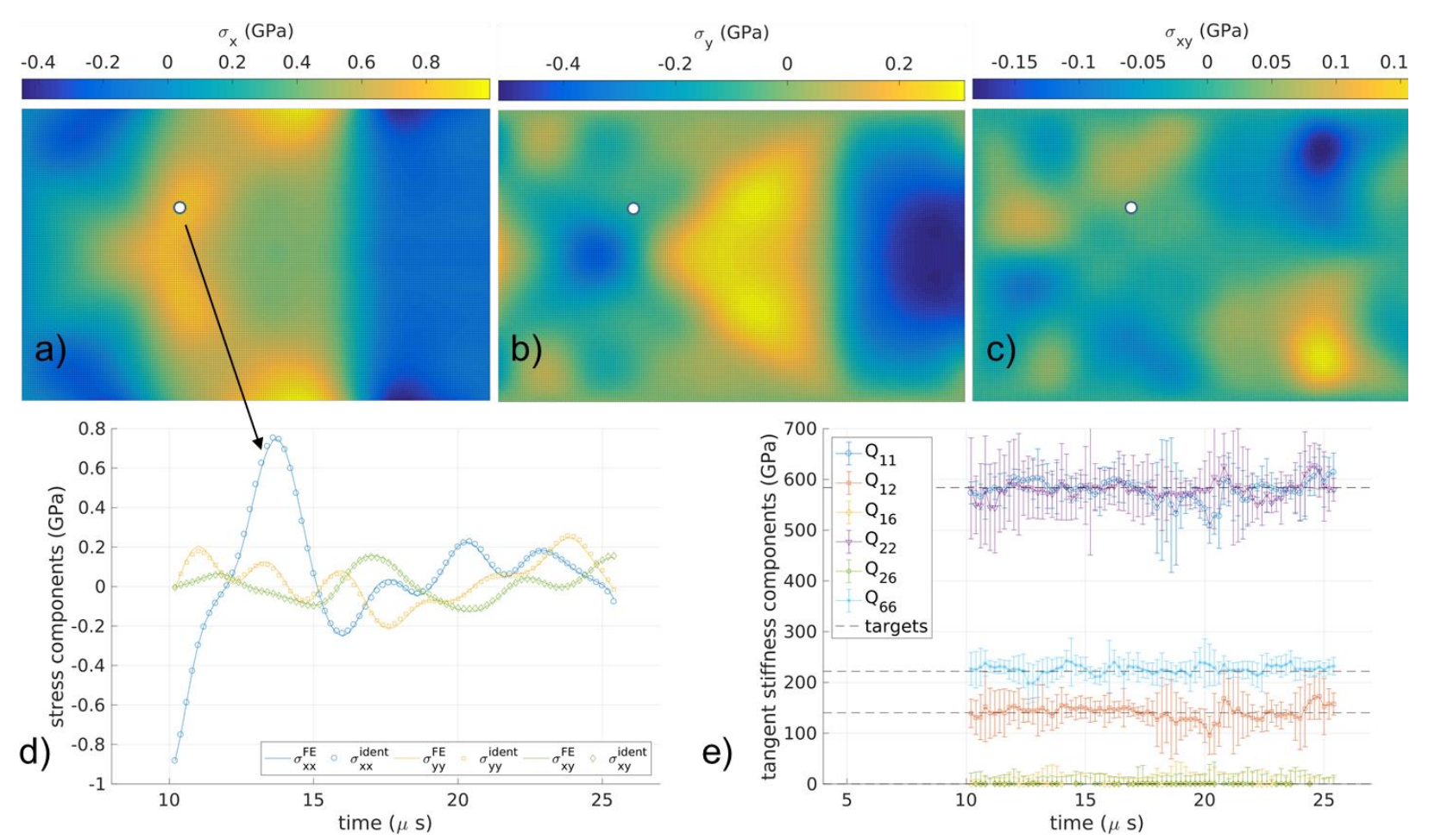

Figure 2: from a to c, identified stress fields at 16 $\mu$ s. d) FE vs reconstructed stress at a specific point over time, and e) identified material properties over time

\section{Conclusion}

Such preliminary results validate the methodology, and evidence that an Image-Based full-field Stress Reconstruction is actually possible in a dynamic context, taking advantage of inertia effects. Some experimental data, on isotropic and orthotropic material, are currently under process and will allow confirming such results in real conditions. Especially, it is planned to capture local fracture strength of materials subjected spalling tests.

\section{References}

[1] Pierron, F. and Forquin, P. Ultra-high-speed full-field deformation measurements on concrete spalling specimens and stiffness identification with the virtual fields method. Strain, 48, 5 (2012), 388-405.

[2] Pierron, F., Zhu, H. and Siviour, C. Beyond Hopkinson's bar. Phil. Trans. R. Soc. A., 372, 2023 (2014), 20130195. [3] Seghir, R. and Pierron, F. A novel image-based ultrasonic test to map material mechanical properties at high strainrates. Exp. Mech., 58, 2 (2018), 183-206. 